\title{
THE AIR TRANSPORTATION HUB-AND-SPOKE DESIGN PROBLEM: COMPARISON BETWEEN A CONTINUOUS AND A DISCRETE SOLUTION METHOD
}

\author{
Guina Sotomayor Alzamora ${ }^{1}$, Fernanda M.P. Raupp ${ }^{1 *}$, \\ Nelio D. Pizzolato ${ }^{1}$ and Adilson E. Xavier ${ }^{2}$
}

Received December 14, 2012 / Accepted September 3, 2013

\begin{abstract}
The hub-and-spoke network design problem, also known as the hub location problem, aims to find the concentration points in a given network flow so that the sum of the distances of the linkages is minimized. In this work, we compare discrete solutions of this problem, given by the branch-and-cut method applied to the $p$-hub median model, with continuous solutions, given by the hyperbolic smoothing technique applied to a min-sum-min model. Computational experiments for particular instances of the Brazilian air transportation system, with the number of hubs varying from 2 to 8 , are conducted with the support of a discretization heuristic and the Voronoi diagram.
\end{abstract}

Keywords: hub location problem, $p$-median, hyperbolic smoothing technique.

\section{INTRODUCTION}

Air freight has grown faster in the past few decades (Bowen, 2004). Particularly in Brazil, about 179 million trips were processed in 2011, $15 \%$ more than in the previous year (INFRAERO, 2011). This growth calls for a rationalization of the air transport system. In this context, there are challenges to be faced in serving this growing demand. The most important challenge appears to be the poor airport infrastructure that prevails in Brazil, where the busiest airports face huge problems of congestion and most do not have physical space available for expansion. A useful strategy to improve the configuration of air transportation systems is the hub-and-spoke network design.

According to Alumur \& Kara (2008), hubs are special facilities that serve as switching, transshipment and sorting points in many-to-many distribution systems. Then, the word hub can be

\footnotetext{
*Corresponding author

${ }^{1}$ Industrial Engineering Department, Pontifical Catholic University of Rio de Janeiro, Rua Marquês de São Vicente, 225, Gávea, RJ, Brazil. E-mails: guinas@gmail.com; fraupp@puc-rio.br; ndp@puc-rio.br

2 Systems Engineering and Computer Science, Federal University of Rio de Janeiro, Centro de Tecnologia Bloco H Sala 319, Cidade Universitária, RJ, Brazil. E-mail: adilson@ cos.ufrj.br
} 
described as a center of importance or interest and the word spoke can be defined as a link or arc. Therefore, the hub-and-spoke system can be interpreted as relevant centers connected to many nodes through links. This strategy aims to optimize the number/distances of linkages in the network, diminishing the overall costs. In this sense, the hub-and-spoke network design problem, also known as the hub location problem, aims to find the centers that are linked to each other, which in turn concentrate regional interconnected nodes that enable minimization of the flow costs (Fulco, 2006).

Just as for the most facility location problems, the generic hub location problem is also known as the network design problem (Campbell \& O'Kelly, 2012). Contreras \& Fernandez (2011) show a review of combined network design and location problems, in which can be observed that the corresponding mathematical models are in general variations of the classic $p$-median model. In particular, the Steiner tree problem can be viewed as a hub location problem where the Steiner points that should be determined are hubs to be located. In this way, the Steiner problem is traditionally and quite logically viewed as a network design problem, but not a location problem (Campbell \& O'Kelly, 2012).

As in Pizzolato et al. (2012), the formal study of hub locations was introduced by O'Kelly (1987) who provided a quadratic programming model for the hub location problem, and proposed two enumeration-based heuristics to solve it. The modeling design considered as assumption that: the number of hubs $p$ was known a priori, no limit on the number of spokes assigned to a hub, each spoke was assigned to a single hub, and all hubs were interconnected.

Indeed, the hub location problem has attracted the attention of researchers from a wide variety of science fields, such as Geography, Operations Research, Transportation (of passengers and of cargo), Telecommunications, among others. An overview of the academic research on hub location problems can be found in Alumur \& Kara (2008), Hekmatfar \& Pishvaee (2009) and Campbell et al. (2009), where can be verified that many sophisticated models have been proposed. Also, some reflections on the origins of the hub location research, comments about its the present status and suggestions for some promising directions for the next 25 years can be found in (Campbell \& O’Kelly, 2012).

In this study, we deal with the hub-and-spoke design of the Brazilian air transportation system, aiming to compare a discrete and a continuous solution method. To this end, the bi-dimensional hub location problem is treated in a simplest way, that is: the only considered parameters of the original problem are the geographical coordinates (latitude and longitude) of the Brazilian airports. No costs are considered in this study.

First, we solve the hub location problem by formulating it as the $p$-hub median model, using the same analogy made by Campbell (1996), where a demand point in a $p$-median model is analogous to an origin-destination pair in the hub-and-spoke problem. The resulting model is an integer linear programming problem whose instances are solved by the branch-and-cut method. 
Then, we consider the application of the hyperbolic smoothing technique, introduced by Xavier (1996), to solve the minimization of the sum of minimal spoke distances, a continuous formulation that models the hub location problem. In general terms, this technique solves a sequence of low dimensional differentiable unconstrained continuous optimization subproblems whose solutions gradually approach a solution of the original problem. This method has been applied to solve other optimization problems such as the determination of spatial molecular structure (Souza et al., 2011) and the determination of radio telecommunication bases (Brito \& Xavier, 2006), for example.

We observe that the discrete method chooses as hub locations the points (nodes) over the network, whereas the solutions of the continuous method can be points out of the network (not nodes), but in between the given network points. So, in other to compare both methods in respect to the minimization of the spoke-distances, we apply a discretization procedure to approximate a point outside the network, given by the continuous method, to the nearest point over the network that results in lower spoke-distances, whenever it is needed.

Computational experiments are conducted with a network of 41 airports of the Brazilian air transportation system, extract from the network of 135 airports built by Figueiredo et al. (2012). The solutions of both methods are compared, varying from 2 to 8 the number of hubs, with the support of the Voronoi diagrams. As suggested by Costa et al. (2010), the number of hubs in the Brazilian air transportation network should not be greater than 10 , due to the adequate degree of clustering in the network.

The paper is organized as follows. As the hub location problem has been already introduced, in Section 2 we briefly describe the $p$-hub median model. Section 3 presents the hyperbolic smoothing technique which is applied to solve a min-sum-min continuous model. Section 4 presents the proposed discretization procedure and the numerical experiments with both methods and with the auxiliary of the Voronoi diagram, while Section 5 outlines the conclusions.

\section{THE $p$-HUB MEDIAN APPROACH}

Likewise Campbell (1996), we formulate the hub location problem as the $p$-median model, where a demand point is interpreted as an origin-destination pair of the hub-and-spoke network, in such a way that the model is now called the $p$-hub median. So, in this approach, the location problem seeks $p$-medians or $p$ concentration points to locate hubs in order to minimize the system impedance, that is, the sum of the spoke-distances.

In the following, we briefly describe the $p$-hub median model. Let $S$ be the set of $n$ distinct points of a given network in the bi-dimensional space; $i \in S$ a given point; $j \in S$ a potential hub median; $p$ the given number of hubs to be located; $\left[d_{i j}\right]_{n \times n}$ the known symmetric distance matrix, where $d_{i j}$ is the distance from point $i$ to potential hub $j$, with $d_{i i}=0, \forall i ;\left[x_{i j}\right]_{n \times n}$ the allocation matrix, where $x_{i j}=1$ if point $i$ is connected to hub $j$ and $x_{i j}=0$ otherwise; and $x_{j j}=1$ if $j$ is a hub median, and $x_{j j}=0$, otherwise. 
Assuming by hypothesis that any point can be chosen as hub, the $p$-hub median model is formulated as follows:

$$
\begin{array}{ll}
\operatorname{minimize} & \sum_{i \in S} \sum_{j \in S} d_{i j} x_{i j} \\
\text { subject to } & \sum_{j \in S} x_{i j}=1, \quad i \in S \\
& \sum_{j \in S} x_{j j}=p \\
& x_{i j}-x_{j j} \leq 0, \quad i, j \in S \\
& x_{i j} \in\{0,1\}, \quad i, \quad j \in S,
\end{array}
$$

where the objective function (1) indicates the minimization of the total distances between the given points and the points selected to be hubs; the constraint set (2) indicates that each point $i$ is connected to only one hub $j$; the constraint (3) guarantees that there exist exactly $p$ located hubs; the constraints (4) state that a given point must be connected to a hub, if it is not a hub itself, and finally the constraint set (5) imposes binary decision variables.

Notice that instead of formulating the hub location problem the model (1)-(5) can be used to formulate the clustering problem, where the $p$ clustering points cover/concentrate the remaining $n-p$ points.

\section{THE HYPERBOLIC SMOOTHING APPROACH}

Let $S=\left\{s_{1}, \ldots, s_{n}\right\}$ denote the set of $n$ given distinct points in the bi-dimensional space to be clustered into a given number $p$ of unknown distinct points. Let $x_{i}, i=1, \ldots, p$, be bidimensional space position of the $p$ potential hubs. In this approach, the hub location problem is formulated as a continuous min-sum-min model as follows:

$$
\begin{array}{ll}
\operatorname{minimize} & \sum_{j=1}^{n} z_{j} \\
\text { subject to } & z_{j}=\min _{i=1, \ldots, p}\left\|s_{j}-x_{i}\right\|_{2}, \quad j=1, \ldots, n,
\end{array}
$$

where the objective function is the sum of each minimum distance between a given point and a potential hub.

So, in order to obtain a convex and differentiable version of the above model, we proceed with the hyperbolic smoothing technique, proposed by Xavier (1996), which applies a sequence of transformations to the above model. Let us first consider the relaxation of the constraints in (6), getting:

$$
\begin{array}{ll}
\operatorname{minimize} & \sum_{j=1}^{n} z_{j} \\
\text { subject to } & z_{j}-\left\|s_{j}-x_{i}\right\|_{2} \leq 0 \quad j=1, \ldots, n, \quad i=1, \ldots, p .
\end{array}
$$


Now, in order to obtain the desired equivalence between (6) and (7), the function $\psi: \mathbb{R} \rightarrow \mathbb{R}$, defined as $\psi(y)=\max \{0, y\}$, is introduced into the inequalities, obtaining the new formulation:

$$
\sum_{i=1}^{q} \psi\left(z_{j}-\left\|s_{j}-x_{i}\right\|_{2}\right)=0, \quad j=1, \ldots, n .
$$

With (8) instead of the constraint set in (7), we still have an undesirable formulation, since the problem has no lower bound. To overcome this shortcoming, a given perturbation parameter $\varepsilon>0$ is introduced, resulting in the bounded but not differentiable optimization problem:

$$
\begin{array}{ll}
\text { minimize } & \sum_{j=1}^{n} z_{j} \\
\text { subject to } & \sum_{i=1}^{p} \psi\left(z_{j}-\left\|s_{j}-x_{i}\right\|_{2}\right) \geq \varepsilon, \quad j=1, \ldots, n .
\end{array}
$$

Now, given the parameter $\tau>0$, let us consider the hyperbolic function $\phi: \mathbb{R} \rightarrow \mathbb{R}$, defined as $\phi(y, \tau)=\left(y+\sqrt{y^{2}+\tau^{2}}\right) / 2$. Thus, the smoothness of model (9) is obtained by replacing $\psi(y)$ by $\phi(y, \tau)$, and also by replacing the norm function by the function $\theta: \mathbb{R}^{n} \times \mathbb{R}^{p} \times \mathbb{R}_{+} \rightarrow$ $\mathbb{R}_{+}$, defined as $\theta\left(s_{j}, x_{i}, \gamma\right)=\sqrt{\left(s_{j}^{1}-x_{i}^{1}\right)^{2}+\left(s_{j}^{2}-x_{i}^{2}\right)^{2}+\gamma^{2}}$ for a given parameter $\gamma>0$, which completes the smoothness procedure, and generates the following problem:

$$
\begin{array}{ll}
\operatorname{minimize} & \sum_{j=1}^{n} z_{j} \\
\text { subject to } & \sum_{i=1}^{p} \phi\left(z_{j}-\theta\left(s_{j}, x_{i}, \gamma\right), \tau\right) \geq \varepsilon, \quad j=1, \ldots, n .
\end{array}
$$

As the functions of the constraints of problem (10) are monotonically increasing in the variable $z_{j}, j=1, \ldots, n$, (Xavier, 2011), they will be active at the optimal solution, and thus problem (10) is equivalent to the following problem:

$$
\begin{array}{ll}
\operatorname{minimize} & \sum_{j=1}^{n} z_{j} \\
\text { subject to } & h_{j}\left(x, z_{j}\right)=\sum_{i=1}^{p} \phi\left(z_{j}-\theta\left(s_{j}, x_{i}, \gamma\right), \tau\right)-\varepsilon=0, \quad j=1, \ldots, n .
\end{array}
$$

Observe that model (11) has a separable structure, since each auxiliary variable $z_{j}$ appears only in one equality constraint. Therefore, as the partial derivative of $h\left(x, z_{j}\right)$ with respect to $z_{j}, j=1, \ldots, n$, is not equal to zero, it is possible to apply the results of the Implicit Function Theorem to compute each component $z_{j}, j=1, \ldots, n$, as a function of the hub location 
variables $x_{i}, i=1, \ldots, p$. In this way, problem (11) is rewritten as the unconstrained optimization problem

$$
\operatorname{minimize} f(x)=\sum_{j=1}^{n} z_{j}(x)
$$

where each $z_{j}(x)$ results from the computation of the single zero of each constraint equation in (11), because in each sum term $\phi$ is strictly increasing with respect to variable $z_{j}$. From the Implicit Function Theorem, the function $z_{j}(x), j=1, \ldots, n$, has derivative with respect to the variable $x_{i}, i=1, \ldots, p$, and so it is possible to compute the gradient of the objective function of problem (12) as

$$
\nabla f(x)=\sum_{j=1}^{n} \nabla z_{j}(x)
$$

where

$$
\nabla z_{j}(x)=-\nabla h_{j}\left(x, z_{j}\right) /\left(\frac{\partial h_{j}\left(x, z_{j}\right)}{\partial z_{j}}\right) .
$$

Summing up, the solution of the hub location problem can be obtained by using an algorithm which solves an infinite sequence of continuous optimization subproblems formulated as (12), where the positive parameters $\varepsilon, \tau$, and $\gamma$ are gradually reduced to zero, just as a smoothing method. Notice that when the algorithm causes $\tau$ and $\gamma$ to approach 0 , the constraints of the subproblems given in (10) tend to those of (9). Also, when the algorithm causes $\varepsilon$ to approach 0 simultaneously, the solution of problem (9) gradually approaches the solution of the original hub location problem (6). Additionally, each unconstrained subproblem (12) can be solved by any method based on first order derivative information.

\section{VORONOI DIAGRAM TECHNIQUE}

Proposed originally by Voronoi, in the middle of the nineteenth century, the Voronoi diagram is a special type of metric space partition determined by Euclidean distances from a set of specified seed points in the bi-dimensional space. This partition with at least 2 seed points results in convex bi-dimensional polygons such that each polygon contains exactly one of these seed points, as well as it contains interior points that are closer to this seed point than any other seed point.

Resembling Boots \& South (1997), the ordinary Voronoi diagram can be defined as follows. Let $\left\{x_{1}, x_{2}, \ldots, x_{p}\right\}$ be the set of $p$ known distinct seed points in $\mathbb{R}^{2}$. Let $x$ denote an arbitrary point in $\mathbb{R}^{2}$. As the Euclidean distance between $x$ and $x_{j}, j=1, \ldots, p$, is given by

$$
\left\|x-x_{j}\right\|=\sqrt{\left(x^{1}-x_{j}^{1}\right)^{2}+\left(x^{2}-x_{j}^{2}\right)^{2}},
$$

the region $V\left(x_{j}\right)=\left\{x \in \mathbb{R}^{2}:\left\|x-x_{j}\right\| \leq\left\|x-x_{i}\right\|, j \neq i, i, j=1, \ldots, p\right\}$ is called the ordinary Voronoi polygon of the seed point $x_{j}$. Indeed, $V\left(x_{j}\right)$ contains all the points that are closer to the seed point $x_{j}$ than any other. Moreover, the set $\Gamma(P)=\left\{V\left(x_{1}\right), \ldots, V\left(x_{p}\right)\right\}$ is a partition of $\mathbb{R}^{2}$, called the ordinary Voronoi diagram associated with the set of seed points. 


\section{COMPUTATIONAL EXPERIMENTS}

In this section, we compare the continuous and the discrete solution approaches that address the hub location problem applied to the Brazilian air transportation system. We then present the computational results obtained by the application of the branch-and-cut algorithm to solve the $p$-hub median model (1)-(5) and by the application of the hyperbolic smoothing technique to solve the min-sum-min model (6). From solver CPLEX 11.2 we get the results of the branchand-cut algorithm. The unconstrained minimization subproblems that are generated by the hyperbolic smoothing technique were coded with Compact Visual FORTRAN, version 6.1, and solved by the BFGS algorithm, a quasi-Newton method from the Harwell Library, available at http://www.cse.scitech.ac.uk/nag/hsl/. The Voronoi diagram algorithm was coded with Visual $\mathrm{C}++$, version 9.0, using Qt and CGAL tools. In addition, the numerical experiments have been carried out on a PC Intel Celeron with 2.7 GHz CPU and 512MB RAM. The compiler was G++ under Windows operational system.

The test instances have been extracted from a hub location study involving 135 airports in the Brazilian air transportation system, built by Figueiredo et al. (2012). Here, we considered a total of 41 airports (nodes) in the air transportation network. The geographic coordinates (latitude and longitude) of each airport were given, and the distances between each pair of airports were computed.

Moreover, the test instances considered the number of hubs varying from 2 to 8 . As suggested by Costa et al. (2010), the number of hubs in the Brazilian air transportation network should not be greater than 10 , due to the adequate degree of clustering in the network. In the cited work, the authors stated that only 4 hubs are necessary for the Brazilian air transportation system.

Regarding the solutions obtained by the smoothing hyperbolic approach, which might be outside the network, we applied a heuristic procedure, called Nearest Allocation (NA), implemented in $\mathrm{C}++$ language, to assign the outsider solution point to the nearest point over the network with lower spoke distances. Figure 1-(a) shows an example of two solution points (hub locations) given by the hyperbolic smoothing technique in light-gray color and four points over the network in dark-gray color. As we can see, there are two hub location points outside the network. Thus, the NA heuristic procedure first locates the hubs at the corresponding nearest points over the network, as we can see in Figure 1-(b). A new allocation of the links to the nearest hub is done by the NA procedure, if the current spoke-distances are greater than the new spoke-distances associated to the nearest hub, as we can see in Figure 1-(c).

Table 1 shows the hub location problem solutions for $p=2, \ldots, 8$ hubs out of the 41 main Brazilian airports, which are candidates to locate hubs, for the plain hyperbolic smoothing approach (HS) and for the hyperbolic smoothing approach combined with the NA heuristic procedure (HSNA), as well as for the $p$-hub median approach.

For the fixed number of hubs $p=4$, Table 2 compares the results of the plain hyperbolic smoothing (HS), the hyperbolic smoothing combined with the NA procedure (HSNA) and the $p$-hub median approaches. These results show that HS has located hubs at points that are not airports. 
We notice that the airports set to locate hubs coincide for the HSNA approach and for the $p$-hub median approach.

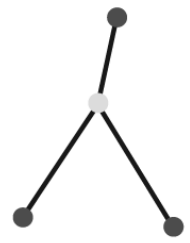

(a)

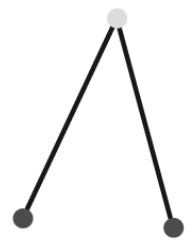

(b)

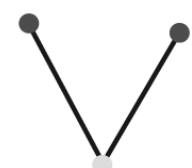

(c)

Figure 1 - Nearest allocation procedure: (a) initial solution, (b) nearest assignment, and (c) nearest assignment with lower spoke-distances.

Table 1 - Results of the hyperbolic smoothing and the $p$-hub median for $p=2, \ldots, 8$.

\begin{tabular}{|c|c|c|c|}
\hline \multirow{2}{*}{ number of hubs } & \multicolumn{3}{|c|}{ Optimal value } \\
\cline { 2 - 4 } & HS & HSNA & $p$-hub median \\
\hline 2 & 300.130 & 302.413 & 300.413 \\
\hline 3 & 220.605 & 223.94 & 223.940 \\
\hline 4 & 184.992 & 185.897 & 185.897 \\
\hline 5 & 158.028 & 159.592 & 159.252 \\
\hline 6 & 139.437 & 140.663 & 140.663 \\
\hline 7 & 126.723 & 128.434 & 127.624 \\
\hline 8 & 114.353 & 115.396 & 115.064 \\
\hline
\end{tabular}

Table 2 - Results for the 4-hubs network design.

\begin{tabular}{|c|c|c|}
\hline Approach & Optimal value & Hubs \\
\hline HS & 184.992 & not airports \\
HSNA & 185.897 & IMP, MAO, MCZ, VCP \\
$p$-hub median & 185.897 & IMP, MAO, MCZ, VCP \\
\hline
\end{tabular}

In Figure 2, the Voronoi diagram refers to the seeds of the 4-hub median approach. In the diagram, the four resulting points given by the plain hyperbolic smoothing technique are showed in light-gray color with their corresponding spokes. As we can see, there are only two points with light-gray color, because the other two (the MAO and the VCP airports) coincide with the medians already found. 


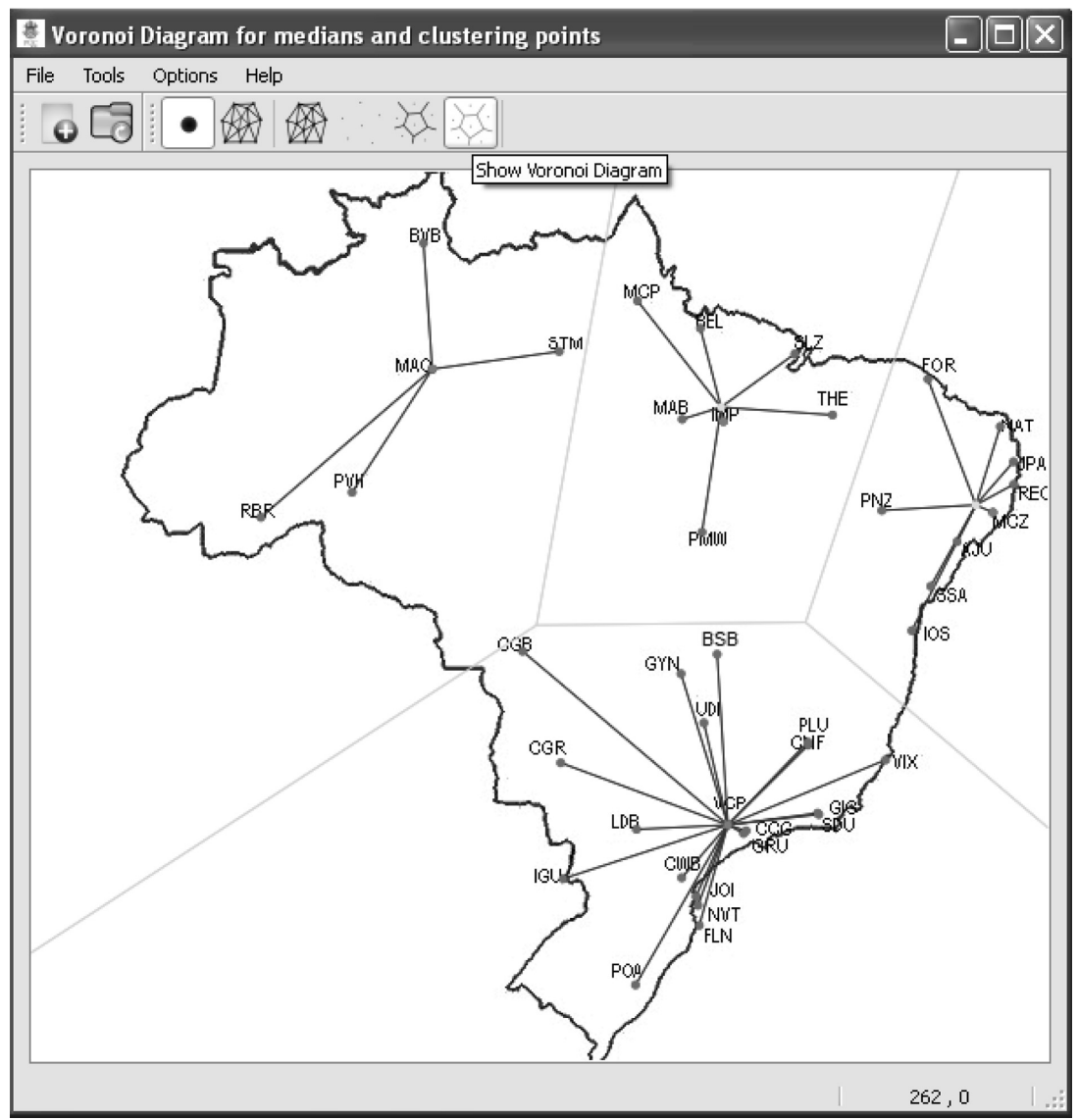

Figure 2 - Brazilian map for 4 hubs as seeds of the Voronoi diagram.

For the fixed number of hubs $p=6$, Table 3 shows the results for the plain hyperbolic smoothing (HS), the hyperbolic smoothing combined with the NA procedure (HSNA) and the $p$-hub median approaches. We notice that the airports set to locate the hubs coincide for the HSNA approach and for the $p$-hub median approach. Here, there is an example of the benefit of applying the NA discretization procedure. From HS, the VCP airport is linked to a hub point that is not an airport. With the application of the NA procedure, VCP is linked first to GIG hub, but the lower total distances is achieved when VCP is linked to JOI hub.

Table 3 - Results for the 6-hubs network design.

\begin{tabular}{|c|c|c|c|}
\hline Approach & Optimal Value & Hubs & Differences \\
\hline HS & 139.437 & not airports & VCP not linked to JOI \\
HSNA & 140.663 & IMP, MAO, JOI, MCZ, GYN, GIG & VCP linked to JOI \\
$p$-median & 140.663 & IMP, MAO, JOI, MCZ, GYN, GIG & VCP linked to JOI \\
\hline
\end{tabular}


In Figure 3, the Voronoi diagram refers to the seeds of the 6-hub median approach. In the diagram, the six resulting points found by the plain hyperbolic smoothing technique are showed with light-gray color with their corresponding spokes. As we can see, there are only four points with light-gray color, because the other two (the GYN and the MAO airports) coincide with the medians already found.

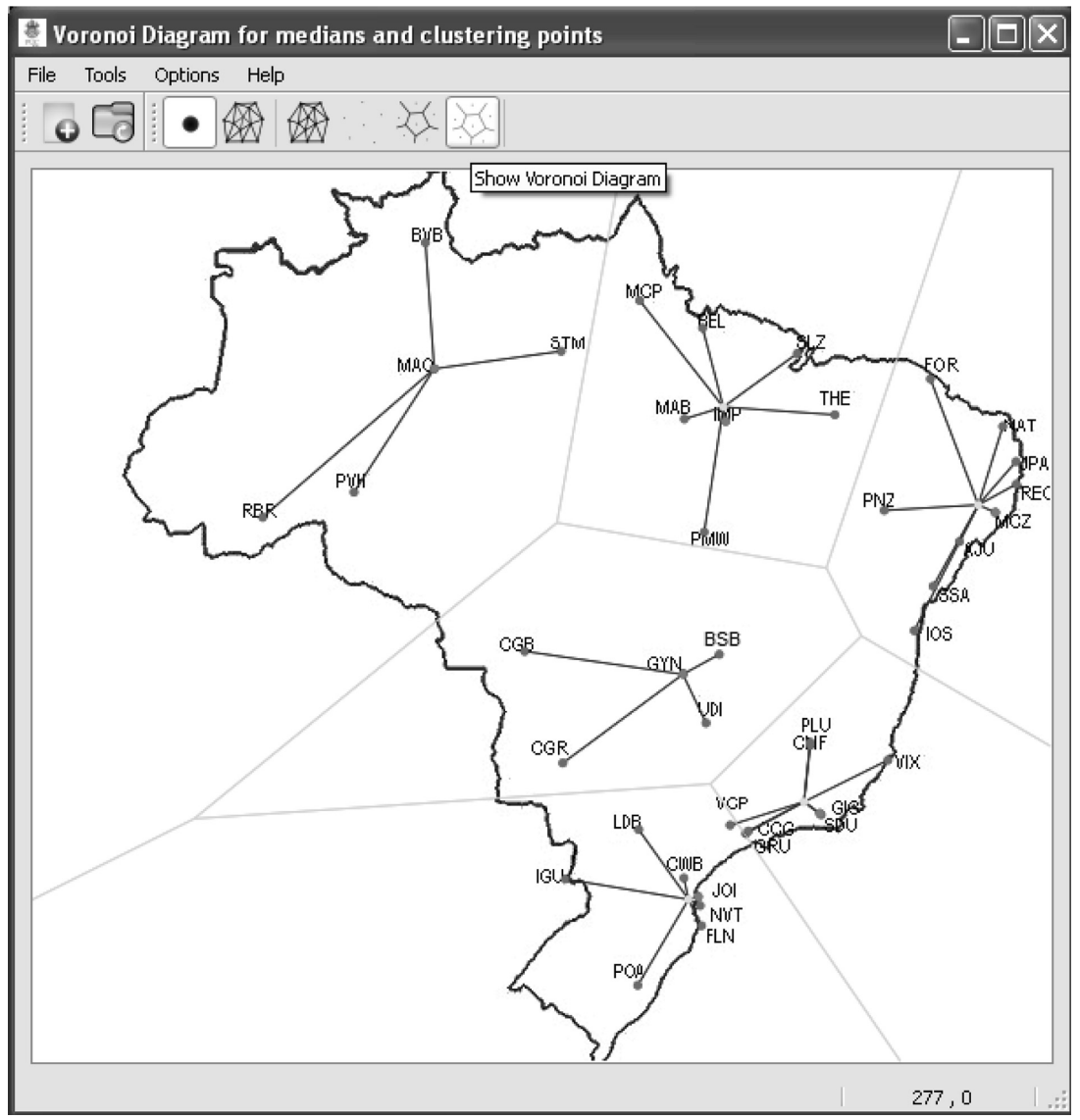

Figure 3 - Brazilian map for 6 hubs as seeds of the Voronoi diagram.

Table 4 - Results for the 8-hubs network design.

\begin{tabular}{|l|c|c|c|}
\hline Approach & Optimal Value & Hubs & Differences \\
\hline HS & 114.353 & not airports & MCP not linked to STM \\
HSNA & 115.396 & MAO, IMP, GYN, GIG, JOI, SSA, JPA, PVH & MCP linked to IMP \\
$p$-median & 115.064 & STM, IMP, GYN, GIG, JOI, SSA, JPA, PVH & MCP linked to STM \\
\hline
\end{tabular}

For the fixed number of hubs $p=8$, Table 4 shows the results for the plain hyperbolic smoothing (HS), the hyperbolic smoothing combined with the NA procedure (HSNA) and the $p$-hub median approaches. We notice that the airports set to locate the hubs does not coincide for the 
HSNA approach and for the $p$-hub median approach. While the $p$-hub median approach chooses STM as median, the HSNA approach chooses MAO, which causes the differences in the covered regions of the hubs, that is, MCP is linked to IMP for HSNA, whereas MCP is linked for STM for the $p$-hub median approach.

In Figure 4, the Voronoi diagram refers to the seeds of the 8-hub median approach. In the diagram, the eight resulting points found by the plain hyperbolic smoothing technique are showed with light-gray color with their corresponding spokes. As we can see, there are only six points with light-gray color, because the other two (the GYN and the SSA airports) coincide with the medians already found.

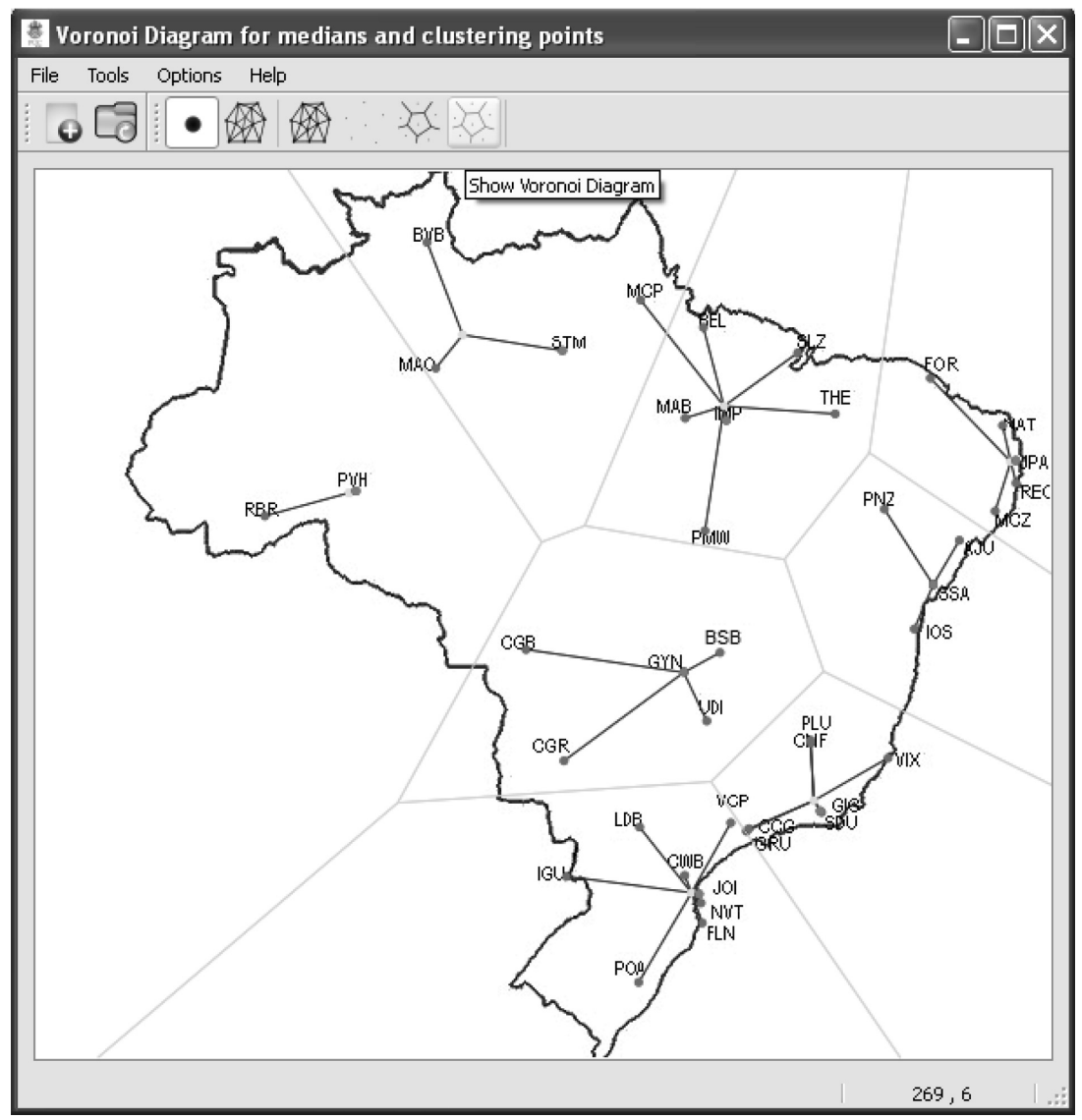

Figure 4 - Brazilian map for 8 hubs as seeds of the Voronoi diagram.

\section{CONCLUSIONS}

Here, we compared the results of two distinct solution methods with the support of a proposed discretization heuristic and the Voronoi diagram to design an extract of the Brazilian air transportation system by introducing hubs. 
Considering instance tests with 41 Brazilian airports and fixed number of hubs varying from 2 to 8 , the solutions of the discrete $p$-hub median approach and the continuous hyperbolic smoothing approach combined with the proposed discretization heuristic were compared in terms of the total spoke-distances. The Voronoi diagram helped to identify the scope of the hubs found by these approaches.

From the computational results, we verified that, with the application of the proposed discretization heuristic, the locations of the hubs are identical when the number of hubs are equal to 4 and 6.

We notice that when the existing airport's infrastructure should be considered, it is more adequate to use a discrete method to determine the locations of the hubs that minimize the total flow distances. With this knowledge, investments to improve the infrastructure of the hubs can then be target. On the other hand, when the infrastructure should be developed, it is more adequate to use a continuous method, because, from the obtained computational results, we verified that the respective optimal total distances is smaller for all instances.

In future work, we plan to consider costs related to the hub locations.

\section{REFERENCES}

[1] Alumur S \& KARA BY. 2008. Network hub location problems: the state of the art. European Journal of Operational Research, 190(1): 1-21.

[2] Bоотs B \& South R. 1997. Modeling retail trade areas using higher-order, multiplicatively weighted voronoi diagrams. Journal of Retailing, 73: 519-536.

[3] BowEN JT. 2004. The geography of freighter aircraft operations in the pacific basin. Journal of Transport Geography, 12(1): 1-11.

[4] BRito JAM \& XAVIER AE. 2006. Modelagens min-max-min para o problema de localização de estações de rádio base. Pesquisa Operacional, 26(2): 295-319.

[5] Campbell JF. 1996. Hub location and the $p$-hub median problem. Operations Research, 44(6): 923-935.

[6] Campbell JF, Ernst A \& Krishnamoorthy M. 2009. Hub Location Problems, chapter 12, pages 373-407. Springer.

[7] Campbell JF \& O’ Kelly ME. 2012. Twenty-five years of hub location research. Transportation Science, 46: 153-169.

[8] Contreras I \& Fernandez E. 2011. General network design: A unified view of combined location and network design problems. European Journal of Operational Research, 219: 680-697.

[9] Costa TFG, Lohmann G \& Oliveira AVM. 2010. A model to identify airport hubs and their importance to tourism in Brazil. Research in Transportation Economics, pages 3-11.

[10] Figueiredo RMA, O' Kelly ME \& Pizzolato ND. 2012. A two stage hub location model for air transportation on Brazil. To appear in International Transactions in Operational Research (ITOR).

[11] FULCO R. 2006. A study of one hub in the brazilian market of air transportation. 
[12] Hekmatfar M \& Pishvaee M. 2009. Hub Location Problem, chapter 11, pages 243-270. PhysicaVerlag Heidelberg.

[13] INFRAERO. 2011. Empresa Brasileira de Infraestrutura Aeroportuária. Internet. www.infraero.gov.br/images/stories/Estatistica/2011/Dez.pdf.

[14] O'KeLLY ME. 1987. A quadratic integer program for the location of interacting hub facilities. European Journal of Operational Research, 32(3): 393-404.

[15] Pizzolato ND, Raupp FMP \& Alzamora GS. 2012. Revision of challenges applied in location problem based in $p$-median models and variants. PODES, Pesquisa Operacional para o Desenvolvimento, 4(1): 13-42 (in portuguese).

[16] Souza M, Xavier AE, Lavor C \& Maculan N. 2011. Hyperbolic smoothing and penalty techniques applied to molecular structure determination. Oper. Res. Lett., 39(6): 461-465.

[17] XAVIER AE. 1996. Hyperbolic penalty: a new method for nonlinear programming with inequalities. International Transactions in Operational Research, 8: 659-671.

[18] XAVIER VL. 2011. Solving the clustering problem by minimizing the sum of distances. M.Sc. thesis, COPPE - UFRJ (in portuguese). 